\title{
Matrix Metalloproteinase-2 as Potential Marker of Early Nephropathy in Type 1 Diabetes
}

\author{
Mohamed Fouad ${ }^{1}$, Maher Boraie ${ }^{2}$ \\ ${ }^{1}$ Nephrology unit, Internal Medicine Departments, Zagazig University Hospital, Zagazig, Egypt \\ ${ }^{2}$ Clinical Pathology Departments, Zagazig University Hospital, Zagazig, Egypt \\ Email address: \\ Dr.M.Fouad@hotmail.com (M. Fouad)
}

To cite this article:

Mohamed Fouad, Maher Boraie. Matrix Metalloproteinase-2 as Potential Marker of Early Nephropathy in Type 1 Diabetes. American Journal of Internal Medicine. Vol. 3, No. 1, 2015, pp. 1-5. doi: 10.11648/j.ajim.20150301.11

\begin{abstract}
Background: Early detection of diabetic nephropathy (DN) is important. Matrix metalloproteinases-2 (MMP-2) regulates a variety of cellular functions including apoptosis and angiogenesis. Diabetic environment stimulates the secretion of MMP-2 that is considered to participate in DN. Objectives: We conducted this study to investigate the level of MMP-2 as a potential marker of early nephropathy in type 1 diabetes. Methods: The total number of the study was 300 participants, among them 100 participants, were healthy volunteers control group with comparable age and sex to other participants (Group 1). The remaining 200 participants were suffering from type 1 diabetes and were categorized according to duration of diabetes into 100 patients had disease duration less than 5 years and all of them non microalbuimnuric (Group 2) and the last 100 patients had disease duration more than 5 years (Group 3). All subjects were submitted to complete clinical examination; routine laboratory investigations, including; random blood sugar (RBS); glycosylated hemoglobin (HbA1C) and quantitative determination of microalbuminuria (MA) for DN. Specific laboratory investigation for MMP-2 by enzyme-linked immunosorbent assay. Results: RBS and HbA-1c were significantly higher in group 3 than group 2. MA significantly detected only in group 3. MMP-2 was significantly higher in group 3 than the other groups 1, 2 and in the meantime significantly higher in group 2 than 1. MMP-2 starts to rise early before the onset of MA in group 2. Eventually duration of diabetes, RBS, HbA1c and MA were positively correlated with the MMP-2 level. ( $\mathrm{r}=0.44 ; \mathrm{P}<0.05),(\mathrm{r}=0.43 ; \mathrm{P}<0.05),(\mathrm{r}=0.58 ; \mathrm{P}<0.05)$ and $(\mathrm{r}=0.71 ; \mathrm{P}<0.001)$ respectively. MMP-2 cutoff level of $\geq 311 \mathrm{ng} / \mathrm{ml}$ had a greater sensitivity and specificity for identifying MA $(\mathrm{P}<0.001)$. Conclusion: MMP-2 level pre-date the clinical evidence of MA, may serve as an important predictor for early development of DN and a potential marker of severity.
\end{abstract}

Keywords: Matrix Metalloprteinase-2, Type1 Diabetes Mellitus, Microalbuminuria, Diabetic Nephropathy

\section{Introduction}

The Diabetes Control and Complications Trial and the U.K. Prospective Diabetes Study have shown that hyperglycemia is an important factor in the development of microvascular complications like nephropathy, retinopathy and peripheral neuropathy in diabetes [1]. One of the possible mechanisms is the accumulation of advanced glycation end products (AGEs), which have been shown to correlate with the degree of diabetic complications [2]. It is widely known that diabetes is associated with an increase in extracellular matrix (ECM), which, because of its slow turnover, is particularly susceptible to AGE accumulation. ECM plays important roles in the regulation of cell function [3]. High glucose concentrations have been shown to act on mesangial cells directly to decrease the activities of matrix metalloproteinases (MMPs), the group of enzymes responsible for mesangium matrix degradation [4] Diabetic nephropathy (DN) is a leading cause of end-stage renal disease, which has a major impact on the disability and high mortality rate seen in patients with both type 1 and type 2 diabetes [5]. Development of diabetic nephropathy is characterized by a thickening of the glomerular basement membrane and expansion of extracellular matrix proteins in the mesangial and tubulointerstitial areas, followed ultimately by progression to glomerular sclerosis and tubulointerstitial atrophy and fibrosis associated with renal dysfunction [6]. Matrix metalloproteinases- 2 and 9 are the most important MMPs in normal kidneys and are therefore assumed to play major roles in basement membrane homeostasis [7]

MMPs are a family of zinc-dependent endopeptidases 
comprising more than 20 members that can degrade various types of ECM components [8]. Among MMPs, matrix MMP-2 also known as $72-\mathrm{kDa}$ gelatinase, constitutively synthesized by fibroblasts, macrophages, epithelial and endothelial cells of mesenchymal origin is the main MMP responsible for degradation of type IV collagen and laminin, major components of ECM proteins [9].The activity of MMP-2 is also regulated by specific tissue inhibitors of MMPs (TIMP-1 and TIMP-2) [10] TIMP-1 is a potent inhibitor of activated MMPs. It is a multifunctional protein, which is found in most tissues and body fluids, and its expression is induced by TGF- $\beta 1$ 1.TIMP-1 appears as a key regulator of extracellular matrix degradation. Moreover, TIMP-1 up-regulates signaling pathways to promote cell cycle progression [11] and in addition to inhibiting MMP-2 and MMP-9 activity, it may inhibit apoptosis in various cell lines [12]

Microalbuminuria is considered to be a marker of risk factor for DN and progressive renal insufficiency in diabetes [13]. However MA may not be as sensitive and specific a predictor of the DN as previously suggested [14]. Other markers of risk for $\mathrm{DN}$ are needed for optimal clinical management so we conducted this study to investigate the level of MMP-2 as a potential marker of early nephropathy in type 1 diabetes.

\section{Patients and Methods}

\subsection{Study Design}

This case-control study was carried out in the Nephrology division at the Zagazig University Hospital, between Jan 2014 to Nov 2014; the aim of the study was explained to all participants and all of them gave informed consent to participate in this study in compliance with the local Institutional Ethics Committee and conformed to the Helsinki Declaration.

Inclusion criterion was patients with type 1 diabetes and patients were excluded from the study if they suffered from other chronic systemic inflammatory or autoimmune disease or malignancy or concurrent use of medications other than insulin (e.g. corticosteroids). Type 2 diabetes patients were also excluded

Demographic information was collected; the total number of the study was 300 participants (150 males and 150 females), among them 100 were healthy volunteers control group (Group 1) with comparable age 17-35 years (y) (25.86 \pm 1.86 y) and sex (48 males and 52 females) to other participants

The remaining 200 participants were suffering from type 1 diabetes and were categorized according to duration of diabetes into farther two subgroups

Group 2; including 100 type 1 diabetic patient (50 males and 50 females); the onset of diabetes was less than 5 years (3.28 $\pm 0.97 \mathrm{y})$, their age ranged from $17-35$ years, $(24.4 \pm 6.69$ y) and all of them were non microalbuminuric.

Group 3; including 100 type 1 diabetic patient (52 males and 48 females); the onset of diabetes was more than 5 years $(8.9 \pm 4.07 \mathrm{y})$ and their age ranged from $18-35$ years $(25.0 \pm 5.06 \mathrm{y})$.

\subsection{Clinical Information}

All participants in this study were subjected to complete clinical examination and all type 1 diabetic patients participating in this study treated with insulin therapy. All participants were instructed how to obtain a fresh, clean first morning urine specimen to exclude orthostatic proteinuria. Microalbuminuria was defined as excretion rate of 30-300 mg /g. urinary creatinine

\subsection{Laboratory Measurements}

All participants were subjected to routine investigations, including liver function tests; alanine aminotransferase (ALT), aspartate aminotransferase (AST), bilirubin, total proteins and albumin and kidney function tests; serum creatinine and blood urea nitrogen and random blood glucose (RBS) on Dimension- $R X L$ Max. Complete blood count on Sysmex Automated Hematology Analyzer KX-21N, Sysmex Corporation, Kobe, Japan. Glycosylated hemoglobin $\left(\mathrm{HbA}_{1} \mathrm{c}\right)$ : Using Ion exchange chromatography followed by quantitative colorimetric determination of glyco-haemoglobin in whole blood, supplied by Stanbio Laboratory, 1261 North Main Street, Boerne, Texas 78006. Urinary microalbumin levels were determined by turbidimetry (Siemens Healthcare Diagnostics, Germany).

\subsection{Measurement of Matrix Metalloproteinase-2}

Venous samples were taken, there was no hemolysis in any of the samples. Immediately after centrifugation, blood serum was cooled on ice, and all samples were stored at $-80^{\circ} \mathrm{C}$ until analysis was performed. The assays for each protein were performed simultaneously to avoid freeze-thaw cycles. Metalloproteinase-2 was measured by enzyme-linked immunosorbent assay (ELISA) technique using kits supplied by RayBiotec, Inc. Catalog number ELH-MMP-2-001.

\subsection{Statistical Methods}

Data were collected, entered and analyzed with an SPSS version19. Data were expressed as mean \pm standard deviation. Analysis of variance (ANOVA or F-test). The correlation between variables is calculated using the Pearson's and the Spearman correlation tests. Predictive values were assessed by the area under the receiver operator characteristic curve. For these tests a probability ( $\mathrm{p}$-value) of $<0.05$ was considered significant.

\section{Results}

\subsection{Demographic Data and Characteristic of Study}

Characteristics of the study and the mean values of all parameters were outlined in table 1. There were non-significant difference in between the three groups regarding their age and sex, while the duration of diabetes was intentionally higher in group 3 according to study design.

Precisely RBS and $\mathrm{HbA}-1 \mathrm{c}$ were significantly higher in group 3 than group 2 and certainly than the control group 
$\mathrm{P}<0.05$.

Particularly MA was significantly detected only in group 3 $\mathrm{P}<0.001$, while $\mathrm{S}$ creatinine stay within the normal levels in all groups, but significantly higher in group $3 . \mathrm{P}<0.05$.

Concerning MMP-2, it was significantly higher in group 3 than in groups 1, 2 and significantly higher in group 2 than group $1 \mathrm{P}<0.05$, interestingly MMP-2 started to rise early before the appearance of MA in group 2 and continue to rise with progression of diabetic complication.

Table 1. Summarized demographic and laboratory characteristics of the study

\begin{tabular}{|c|c|c|c|c|}
\hline Parameter & Group 1 (100) & Group 2 (100) & Group 3 (100) & P value \\
\hline Gender $(\mathrm{M} / \mathrm{F})$ & $0.9 / 1$ & $1 / 1$ & $1 / 0.9$ & NS \\
\hline Age (years) & $25.86 \pm 1.86$ & $24.4 \pm 6.69$ & $25.0 \pm 5.06$ & NS \\
\hline Period of diabetes (years) & None & $3.28 \pm 0.97$ & $8.9 \pm 4.07^{\mathrm{b}}$ & $0.001 *$ \\
\hline Creatinine (mg/dl) & $0.64 \pm 0.16$ & $0.76 \pm 0.26$ & $0.95 \pm 0.34^{\mathrm{ab}}$ & $<0.05^{*}$ \\
\hline RBG (mg/dl) & $103 \pm 20.4$ & $167 \pm 51.9^{\mathrm{a}}$ & $215.6 \pm 75.7^{\mathrm{ab}}$ & $<0.05^{*}$ \\
\hline $\mathrm{HbA}-1 \mathrm{c}(\%)$ & $6.1 \pm 1.3$ & $8.42 \pm 1.8^{\mathrm{a}}$ & $9.17 \pm 1.9^{\mathrm{ab}}$ & $<0.05^{*}$ \\
\hline MMP-2 (ng/ml) & $94.4 \pm 21.75$ & $265.99 \pm 45.38^{\mathrm{a}}$ & $598.45 \pm 101.39^{\mathrm{ab}}$ & $<0.001^{*}$ \\
\hline
\end{tabular}

a indicates a significant difference as compared to Group 1

b indicates a significant difference as compared to Group 2

* Significant

\subsection{Correlates of MMP-2 and other Parameters}

All study parameters were correlated with $M M P-2$ level: Age $(\mathrm{r}=0.13$; NS), disease duration $(\mathrm{r}=0.44 ; \mathrm{P}<0.05)$, random blood glucose $(\mathrm{r}=0.43 ; \mathrm{P}<0.05), \mathrm{HbA}_{1} \mathrm{c}(\mathrm{r}=0.58 ; \mathrm{P}<0.05)$ and $\mathrm{MA}(\mathrm{r}=0.71 ; \mathrm{P}<0.001)$. Except age the other parameters were positively correlated with the MMP-2 level.

Table 2. Sensitivity and Specificity of MMP-2

\begin{tabular}{|c|c|c|c|c|c|c|c|c|c|}
\hline & No of patients & & & Sensitivity & Specificity & PPV & NPV & Карра & $\mathbf{P}$ \\
\hline MMP-2 & MA & Without (MA) & Total & \multirow{4}{*}{88.9} & \multirow{4}{*}{80.6} & \multirow{4}{*}{57.1} & \multirow{4}{*}{96.2} & \multirow{4}{*}{0.58} & \multirow{4}{*}{$<0.001$} \\
\hline$\geq 311$ & 40 & 30 & 70 & & & & & & \\
\hline$<311$ & 4 & 126 & 130 & & & & & & \\
\hline Total & 44 & 156 & 200 & & & & & & \\
\hline
\end{tabular}

Demonstrated ROC analysis, MMP-2 cutoff level of $\geq 311 \mathrm{ng} / \mathrm{ml}$ had a greater sensitivity and specificity for identifying MA

\section{Discussion}

Diabetic nephropathy is characterized by accumulation of mesangium matrix and thickening of basement membrane within the glomeruli. These changes are the result of an imbalance in the synthesis and degradation of ECM components, in particular collagen, fibronectin, and laminin. MMP-2 is responsible for degradation of type IV collagen and laminin, major components of ECM proteins [9]. Early detection of diabetic microvascular complication, particularly nephropathy, has a pivotal role in prevention of end-stage renal disease in children and adolescents with diabetes [15]. MA may not be as sensitive and specific a predictor of the DN as previously suggested [14]. The current study was conducted to investigate the level of MMP-2 as a potential marker of early nephropathy in type 1 diabetes.

In the current study, we demonstrated that in contrast to healthy subjects MMP-2 start to rise early in type 1diabetes within the first 5 years from the onset of diagnosis even before the appearance of MA then markedly rises with disease progression and clinical presentation of DN. In addition, we observed that MMP-2 at level $\geq 311$ can predict MA. Such results allow us to postulate that MMP-2 may be an early marker of DN and also a good index of severity. However, these results are generally compatible with other studies which found that MMP-2 is not only elevated in type 1diabetes than healthy subjects, but also found MMPs markedly raises in relation to increased risk of nephropathy [16-20].

Hyperglycemia per se may excuse the previous results as we observed that RBS and HbA-1c were higher in group 3 than group 2 patients and we also observed positive correlation between hyperglycemia reflected by higher RBS and $\mathrm{HbA}-1 \mathrm{c}$ and MMP-2. These observations were confirmed by another study [21].

However hyperglycemia increases the synthesis of many matrix components, change the matrix itself to become more resistant to degradation and increases cross-linking of collagen molecules, making them less susceptible to degradation by proteolytic enzymes. This increase in cross-linking is mediated by accumulation of AGEs proteins, which can also affect MMP expression and activities [22].

As most of the diabetic patients partaking in in this study were uncontrolled, consequently, is the strict control of hyperglycemia improving the MMP-2 level, matrix expansion and minimize microvascular complication?. The answer to this question needs further researches. However, some articles have been demonstrated that the thickness of the basement membrane is increased in subjects with type 1 diabetes, and that such changes can be arrested by improving blood glucose control [23].

We failed to find any correlation between age and MMP-2. This finding was in agreement with another study [24]. On the 
other hand, diseases duration positively correlated with MMP-2 levels and this finding appeared to be logical as duration of diabetes, independently predicts diabetes severity and complications [25]

Contrary to our results, Jacek et al study [26], observed MMP-2 serum levels in type 2 diabetic nephropathy were still lower than those in non-diabetic patients with chronic renal failure. The apparent discrepancy between the two studies may be ascribed to different study populations and characteristic; as the latter study was included different age, type 2 diabetes and compared with non diabetic chronic renal failure patients

MA is a traditional marker of endothelial dysfunction and microvascular complication of DN [27]. Recently MMP-2 also has also been suggested to reflect the microvascular complication of type 1 diabetes [28]. MA may not be as sensitive and specific a predictor of the DN as previously suggested [14], additionally in the current study MMP-2 started to rise early before the appearance of MA and MMP-2 at the level of $\geq 311$ ( 3 folds the healthy control) can predict MA, so it may predict severity

\section{Conclusion}

This study has demonstrated that MMP-2 could be useful as a screening marker for early detection of diabetic nephropathy, may pre-date clinical evidence of MA and a potential marker of severity.

\section{Acknowledgments}

The authors wish to thank the staff of the Clinical pathology and the Nepherology division of Zagazig University Hospital.

\section{References}

[1] DCCT: Implications of the Diabetes Control and Complications Trial. American Diabetes Association. Diabetes.1993; 42:1555-1558,

[2] Monnier VM, Bautista O, Kenny D, Sell DR, Fogarty J, Dahms W, Cleary PA,Lachin J, Genuth S: Skin collagen glycation, glycoxidation, and crosslinking are lower in subjects with long-term intensive versus conventional therapy of type 1 diabetes: relevance of glycated collagen products versus HbAlc as markers of diabetic complications. DCCT Skin Collagen Ancillary Study Group. Diabetes Control and Complications Trial.Diabetes.1999; 48:870-880

[3] Mauch C: Regulation of connective tissue turnover of cell-matrix interactions. Arch Dermatol Res.1998; 290: S30-S36

[4] Nagase H, Woessner JF Jr: Matrix metalloproteinases. J Biol Chem.1999; 274:21491-21494.

[5] Maisonneuve P, Agodoa L, Gellert Ret al. Distribution of primary renal diseases leading to end-stage renal failure in the United States,Europe, and Australia/New Zealand: results from an international comparative study. Am J Kidney Dis.2000; 35: $157-165$
[6] Gilbert RE, Cooper ME. The tubulointerstitium in progressive diabetic kidney disease: more than an aftermath of glomerular injury? Kidney Int.1999; 56: 1627-1637

[7] Furness PN: (1997) Basement membrane synthesis and degradation. J Pathol, 183 (1): 1-3.

[8] Lenz O, Elliot SJ, Stetler-Stevenson WG. Matrix metalloproteinases in renal development and disease. J Am Soc Nephrol.2000; 11: 574-581

[9] McLennan SV, Kelly DJ, Cox AJet al. Decreased matrix degradation in diabetic nephropathy: effects of ACE inhibition on the expression and activities of matrix metalloproteinases.Diabetologia. 2002; 45: 268-27

[10] Hernandez-Barrantes S, Shimura Y, Soloway PD, Sang QA, Fridman R: Differential roles of TIMP-4 \& TIMP-2inpro-MMP-2 activation by MT1-MMP.Biochem Biophys Res Commun.2001; 281:126-130

[11] Akahane T, Akahane M, Shah A, Thorgeirsson UP. TIMP-1stimulates proliferation of human aortic smooth muscle cells and Ras effector pathways. Biochem Biophys Res Commun.2004; 1 : 440-5.

[12] Han X, Sun Y, Scott S, Bleich D. Tissue inhibitor of metalloproteinase-1 prevents cytokine -mediated dysfunction and cytotoxicity in pancreatic islets and beta-cells. Diabetes.2001; 50: 1047-55

[13] American Diabetes Association: Diabetic nephropathy (Position Statement). Diabetes Care.2000; 23 (Suppl. 1): S69-S72

[14] Bahman P, Tabaei M, AL-K assab , Liza L,I lag, Cathernen M, Zawacki RN and William H. Herman. Does Microalbuminuria Predict Diabetic Nephropathy?. Diabetes Care.2001; 24:1560 $-1566$

[15] Zelmanovitz T, Gerchmann F, Balthazar A, Thomazelli F, Matos J, and Canani L, : Diabetic nephropathy: Diabetology and metabolic syndrome. 2009; 1:10.

[16] Sedegheh Gharagozlian, Katja Svennevig, Hans-Jacob Bangstad, JanOlof Winberg and Svein Olav Kolset..MMP in subjects with type 1diabetes BMC; Clinical Pathology.2009; 9:7 doi: $10.1186 / 1472-6890-9-7$

[17] Mai M Y, Hoda A, Sawsan T, Hanaa S, Ola M, Manal A M, Nagham M, B, A., MMP-2 as a marker of microvascular complications in Children and Adolescents with Type 1 Diabetes Mellitus. Macedonian Journal of Medical Sciences.2011; $\quad 15 ; \quad 4 \quad$ (1): $\quad 81-88$ doi:10.3889/MJMS.1857-5773.2011.0151.

[18] Thrailkill KM, Bunn RC, Moreau CS, Cockrell GE, Simpson PM, Coleman HN, Frindik JP, Kemp SF, Fowlkes JL: MMP-2 Dysregulation in Type I Diabetes. Diabetes Care.2007; 30 (9): 2321-2326

[19] Flory B, Glowinska B, urban M, Peczynska J. MMP-2 and MMP-9 and their inhibitors TIMP-1 and TIMP-2 levels in children and adolescents with type 1 diabetes, Edokrynol Diabetol Chor Przemiany Materii Wieku Rozw.2006; 12 (3): 184-189.

[20] Ming-Yuh Shiau, Shih-Tzer Tsai, Kan-Jen Tsai, Ming-Lih Haung, Increased circulatory MMP-2 and MMP-9 levels and activities in patients with type 1 diabetes mellitus. THE MOUNT SINAI JOURNAL OF MEDICINE.2006; Vol. 73 No. 7. 
[21] Symeonidis C(1), Papakonstantinou E, Galli A, Tsinopoulos I, Mataftsi A, Batzios S, Dimitrakos SA.. Matrix metalloproteinase (MMP-2, -9) and tissue inhibitor (TIMP-1, -2) activity in tear samples of pediatric type 1 diabetic patients: MMPs in tear samples from type 1 diabetes. Graefes Arch Clin Exp Ophthalmol.2013; 251 (3): 741-9.

[22] Kanwar K, Wada J, Lin Sun, Ping Xie, Elisabith I, Sumnat C, and Nad Farhad R: Diabetic nephropathy: Mechanisms of renal disease progression: Exp Biol Med.2007; 233:4-11.

[23] Bangstad HJ, Osterby R, Dahl-Jorgensen K, Berg KJ, Hartmann A, Hanssen KF: Improvement of blood glucose control in IDDM patients retards the progression of morphological changes in early diabetic nephropathy. Diabetologia.2007; 37 (5): 483-490.

[24] Zelmanovitz T, Gerchmann F, Balthazar A, Thomazelli F, Matos J, and Canani L, : Diabetic nephropathy: Diabetology and metabolic syndrome. 2009; 1:10.
[25] Sophia Zoungas, Sophia Zoungas, Mark Woodward, Qiang Li, Mark E. Cooper, Pavel Hamet et al. (2014). Impact of age, age at diagnosis and duration of diabetes on the risk of macrovascular and microvascular complications and death in type 2 diabetes. Diabetologia.2014;DOI: 10. $1007 / \mathrm{s} 00125-014$ $-3369-7$

[26] Jacek Rysz, Maciej Banach, Robert A. Stolarek, Jaroslaw Pasnik, et al (2007). MMP-2 and MMP-9 and metalloproteinase tissue inhibitors TIMP-1 and TIMP-2 in DN.JNEPHROL.2007;; 20: 444-452

[27] Messent JWC, Elliott TG\&Hill RD, et al. (1992) Prognostic significance of microalbuminuria in Insulin- dependent diabetes mellitus: A twenty-three year follow-up study. Kidney Int; 41: 836-839

[28] Derosa G, Avanzini MA, Geroldi D, Fogari R, Lorini R, De Silvestri A,et al: (2005) Matrix MMP- 2may be a marker of microangiopathy in children and adolescents with type I diabetes Diabetes Res Clin Pract.; 70 (2): 119-125 